\title{
Use of anabolic-androgenic steroids masking the diagnosis of pleural tuberculosis: a case report Carlos Fernández de Larrea ${ }^{1}$, Aglae Duplat ${ }^{1}$, Ismar Rivera-Olivero ${ }^{2}$ and Jacobus $\mathrm{H}$ de Waard*2
}

Address: ${ }^{1}$ Department of Internal Medicine, Hospital Vargas de Caracas, Venezuela and ${ }^{2}$ Laboratorio de Tuberculosis, Instituto de Biomedicina, Universidad Central de Venezuela, San Nicolas a Providencia, San José, Caracas, Venezuela

Email: Carlos Fernández de Larrea - clarrea@cantv.net; Aglae Duplat - aglaia@cantv.net; Ismar Rivera-Olivero - ismaralejandra@hotmail.com; Jacobus H de Waard* - jacobusdeward@gmail.com

* Corresponding author

Published: 28 January 2009

Journal of Medical Case Reports 2009, 3:30 doi:10.1186/1752-1947-3-30
Received: 15 February 2008

Accepted: 28 January 2009

This article is available from: http://www.jmedicalcasereports.com/content/3/1/30

(C) 2009 de Larrea et al; licensee BioMed Central Ltd.

This is an Open Access article distributed under the terms of the Creative Commons Attribution License (http://creativecommons.org/licenses/by/2.0), which permits unrestricted use, distribution, and reproduction in any medium, provided the original work is properly cited.

\begin{abstract}
Introduction: Tuberculous pleural effusions are not always easy to diagnose but the presence of a lymphocyte-rich exudate associated with an increased adenosine deaminase level and a positive skin test result are highly sensitive diagnostic signs.

Case presentation: We report a case of pleural tuberculosis in a 31 -year-old white male patient from Caracas, Venezuela who was negative for human immunodeficiency virus and presented 2 weeks after injecting the anabolic-androgenic steroid nandrolone decanoate, in whom all the tests for tuberculosis were initially negative; an eosinophilic pleural effusion with a low adenosine deaminase level, a negative tuberculin skin test and negative for acid-fast bacilli staining and culture of the pleural fluid. After excluding other causes of eosinophilic pleural effusion malignant pleural effusion was suspected. The patient did not return until 4 months later. The second thoracentesis obtained a pleural fluid suggestive for tuberculosis, with a predominance of lymphocytes, an elevated adenosine deaminase level $(5 \mathrm{I} \mathrm{U} / \mathrm{I})$ and a positive tuberculin skin test. Culture of pleural fragments confirmed pleural tuberculosis.
\end{abstract}

Conclusion: This case suggests that the use of an anabolic-androgenic steroid masks the definitive diagnosis of pleural tuberculosis by changing the key diagnostic parameters of the pleural fluid, a finding not previously reported. Available evidence of the effects of anabolic steroids on the immune system also suggests that patients using anabolic-androgenic steroids might be susceptible to developing tuberculosis in either reactivating a latent infection or facilitating development of the disease after a recent infection.

\section{Introduction}

The cause of an exudative pleural effusion (EPF) is often difficult to determine, but tuberculosis (TB) must be considered, especially in countries with a high prevalence of TB. The diagnosis of a tuberculous pleural effusion is based on the Ziehl-Neelsen staining for acid-fast bacilli (AFB) and on the growth of Mycobacterium tuberculosis from pleural fluid or biopsy. However, if no AFB are found, cultures take 4-6 weeks to be positive, and therapeutic decisions need to be made before the results are 
available. A positive tuberculin skin test (TST) can be helpful, but may be negative in a third of the patients with tuberculous pleural effusions. An elevated level of the Adenosine Deaminase (ADA) activity in the pleural fluid has proven to be very sensitive and specific for the diagnosis of pleural $\mathrm{TB}$, specially when the differential cell count of an exudative effusion shows a lymphocyte neutrophil ratio of 0.75 or greater $[1,2]$. We report a case of pleural TB in a patient who presented 2 weeks after injecting the androgenic-anabolic steroid (AAS) nandrolone decanoate, in whom all the tests for TB were initially negative. This case report reviews the possible effects of AAS therapy on the immune system and changing important diagnostic parameters in the pleural fluid.

\section{Case presentation}

A 31-year-old white man from Caracas, Venezuela presented in the emergency room complaining of daily evening fever, night sweats, pleuritic chest pain and shortness of breath for the previous 2 weeks. Up until 2-weeks before, over 10 days, he had received a daily dose of intramuscular nandrolone (Deca-Durabolin ${ }^{\circledast} 50 \mathrm{mg}$ ), but was not taking any other medication. A chest X-ray showed a pleural effusion occupying 30\% of the left hemi-thorax. His physical examination, blood count and serum biochemistry were otherwise unremarkable. A thoracentesis obtained clear fluid, exudative by lactate dehydrogenase (LDH) and protein levels, (310 UI/l and 4,8 gr/dl respectively) normal $\mathrm{pH}(7,46)$ and predominantly eosinophils $(30 \%)$ in the leukocyte differential count $(2500$ cell per cubic milliliter) and 50\% lymphocytes and 20\% neutrophils. No peripheral blood eosinophilia was found. AFB staining of the pleural fluid was negative and the ADA level was normal $(21 \mathrm{UI} / \mathrm{L})$. The patient gave no history of TB contact and a TST was negative $(0 \mathrm{~mm})$. Culture of the pleural fluid for bacteria on blood agar and for mycobacteria on Lowenstein Jensen and Stonebrink medium was negative. A parasitic infection was suspected but serial stool examinations were negative for parasites. No definitive diagnosis was made, but malignant pleural effusion was suspected.

After thoracentesis the patient felt better and did not return to the hospital until 4-months later, when he returned to the emergency room with similar pleuritic symptoms, fever and night sweats. Additionally, he had a nonproductive cough and had lost more than 5 kilograms in weight since the previous visit. Examination of the lungs revealed decreased breath sound in the lower left hemi-thorax, but the rest of the physical examination was normal. A chest X-ray again showed the left sided pleural effusion, slightly smaller than on the previous study. A second thoracentesis obtained turbid fluid, $\mathrm{pH} 7 \cdot 41$, 3100 cells per cubic millimeter, $90 \%$ mononuclear and $3 \%$ eosinophils, 5,4 gr/dl proteins and $242 \mathrm{UI} / \mathrm{dl} \mathrm{LDH}$.
Glucose, amylase levels, hematological parameters, renal and hepatic serum tests were normal. The effusion/serum ratio for proteins and LDH were 0.77 and 0.83 respectively. The ADA on the pleural fluid was elevated ( $51 \mathrm{U} / \mathrm{L}$ ) and suggestive of tuberculosis, and the TST was positive $(20 \mathrm{~mm})$. Ziehl-Neelsen staining for AFB was negative both on induced sputum and pleural liquid. A pleural biopsy showed a chronic pleurisy with multiple granulomas with central necrosis, compatible with pleural TB and a culture of the pleural tissue was positive for M. tuberculosis after 4 weeks. The patient's symptoms disappeared after starting treatment with anti-tuberculosis drugs, and the chest X-ray showed resolution of the effusion 4 weeks later, turning completely normal.

\section{Discussion}

Two weeks after anabolic steroid use our patient had a pleural effusion that was predominantly eosinophilic. Pleural fluid eosinophilia is very often related with conditions associated with the presence of blood or air in the pleural space [3] but our patient had no evidence of chest trauma, hemothorax, or pneumothorax. Pulmonary embolism or benign asbestos pleural effusions are other common causes of eosinophilic pleural effusion, but were also excluded, even as a parasitic or bacterial infection [3]. The relationship between symptom onset and initiation of AAS therapy in combination with the pleural fluid eosinophilia raised the suspicion of a drug-induced pleural reaction (reviewed in [4]). Certain drugs (Table 3 in [3]) can cause pleural eosinophilia. However, most of these drugs also cause peripheral eosinophilia which was not found in our patient. In addition, AAS are not listed as drugs that cause eosinophilic pleurisy. Because the frequency of malignant etiology among EPEs in general is high and has varied between $6 \%$ and $40 \%$ in different studies [3] malignancy was suspected. We did not consider a tuberculous pleural effusion as they very rarely contain high numbers of eosinophils. A prevalence of only $1 \cdot 3 \%$ among 700 tuberculous pleural effusions has been reported [5]. In addition, the patient had a negative TST and pleural liquid with a normal ADA value, two important other parameters for the consideration of tuberculous pleurisy $[1,2]$. These three parameters however were strikingly changed at the second visit 4-months later, when the TST was positive, the pleural fluid was predominantly lymphocytic, the ADA level was elevated and tuberculous pleurisy was diagnosed.

Only a few data are available about the effects of anabolic steroids on the immune system and therefore do not allow firm conclusions but we hypothesize that the use of nandrolone could have changed the key parameters of the pleural fluid, and could have played a role in developing tuberculosis in either reactivating a latent infection or facilitating development of disease after a recent infection. 
It has been shown that a high dose of anabolic steroids can have significant effects on immune responses. Nandrolone decanoate directly modifies the cytokine pattern in human and murine models increasing the production of inflammatory cytokines IL-1 beta and TNF-alpha, without affecting IL-2 or IL-10 production but significantly inhibiting IFN gamma production [6] This last cytokine is essential in monocyte and macrophage Th1 activation, the most effective response against intracellular pathogens like $M$. tuberculosis. In addition, IFN gamma is a potent inductor of intracellular ADA [7] and the low level of ADA activity found in the pleural liquid just after the use of nandrolone decanoate could have been caused by the inhibitor effect of the AAS on the IFN production. This model however doesn't explain the high level of eosinophils in the pleural space just after AAS use. An eosinophilic pleural effusion is supposed to be related to IL-5 production [8], and no relation has been described between AAS and IL-5 production to explain the high level of eosinophils in the pleural space.

In conclusion, we believe that the use of AAS should be included when evaluating EPEs and should be considered a possible cause of changing pleural fluid parameters and of developing TB. It is important to stress that, as has been observed for the reactivation of $\mathrm{TB}$ after the use of for example glucocorticosteroids [9], the frequency of developing $\mathrm{TB}$ could be low in countries with a low incidence of TB (for glucocorticosteroids, $0 \%$ in the USA and Greece, $0.6 \%$ in France and $1.35 \%$ in Spain have been reported). In contrast, the frequency of development of TB after the use of glucocorticosteroids was much higher in studies performed in countries with a moderate to high incidence of TB (from $2 \cdot 5 \%$ in South Korea to $13 \cdot 8 \%$ in the Philippines [9]).

\section{Conclusion}

This case suggests that patients using anabolic steroids might be susceptible to developing tuberculosis in either reactivating a latent infection or facilitating development of disease after a recent infection and that the use of nandrolone limits the diagnostic value of key parameters for the diagnosis of pleural $\mathrm{TB}$, a finding not previously reported. We would like to recommend that attention should be paid to the possibility of nandrolone as a drug implicated in tuberculous eosinophilic pleurisy.

\section{Abbreviations}

ADA: adenosine deaminase; TB: tuberculosis; AAS: anabolic-androgenic steroid; EPF: eosinophilic pleural effusion; TST: tuberculin skin test; AFB: acid-fast bacilli; LDH: lactate dehydrogenase.

\section{Consent}

Informed written consent was obtained from the patient for publication of this manuscript.

\section{Competing interests}

The authors declare that they have no competing interests.

\section{Authors' contributions}

$\mathrm{CL}$ and $\mathrm{AD}$ were involved in the case directly, performed the literature search and assisted in the preparation of the manuscript. IRO was involved in the laboratory diagnosis, in the literature review and drafting of the manuscript. JW was involved in drafting the manuscript and in overall supervision. All authors read and approved the final manuscript.

\section{Acknowledgements}

Funding of a LOCTI research grant from Shell Venezuela CA was received for the preparation of this case report.

\section{References}

I. Valdes L, Alvarez D, San Jose E, Penela P, Valle JM, García-Pazos JM, Suárez J, Pose A: Tuberculous pleurisy: a study of 254 patients. Arch Intern Med 1998, I 58:2017-202I.

2. Burgess LJ, Maritz FJ, Le Roux I, Taljaard J]: Combined use of pleural adenosine deaminase with lymphocyte/neutrophil ratio. Increased specificity for the diagnosis of tuberculous pleuritis. Chest 1996, 109:414-419.

3. Kalomenidis I, Light RW: Eosinophilic pleural effusions. Curr Opin Pulm Med 2003, 9:254-260.

4. Huggins JT, Sahn SA: Drug-induced pleural disease. Clin Chest Med 2004, 25: I4I-I 53.

5. Adelman M, Albelda SM, Gottlieb J, et al:: Diagnostic utility of pleural fluid eosinophilia. Am J Med 1984, 77:915-920.

6. Hughes TK, Fulep E, Juelich T, Smith EM, Stanton GJ: Modulation of immune responses by anabolic androgenic steroids. Int J Immunopharmaco 1995, 17:857-863.

7. Murray JL, Mehta K, Lopez-Berestein G: Induction of adenosine deaminase and $5^{\prime}$ nucleotidase activity in cultured human blood monocytes and monocytic leukemia (THP-I) cells by differentiating agents. J Leukoc Biol 1988, 44:205-2II.

8. Kalomenidis I, Light RW: Pathogenesis of the eosinophilic pleural effusions. Curr Opin Pulm Med 2004, 10:289-293.

9. Falagas ME, Voidonikola PT, Angelousi AG: Tuberculosis in patients with systemic rheumatic or pulmonary diseases treated with glucocorticosteroids and the preventive role of isoniazid: a review of the available evidence. Int J Antimicrob Agents 2007, 30:477-486.

Publish with Biomed Central and every scientist can read your work free of charge

"BioMed Central will be the most significant development for disseminating the results of biomedical research in our lifetime. "

Sir Paul Nurse, Cancer Research UK

Your research papers will be:

- available free of charge to the entire biomedical community

- peer reviewed and published immediately upon acceptance

- cited in PubMed and archived on PubMed Central

- yours - you keep the copyright

Submit your manuscript here:

http://www.biomedcentral.com/info/publishing_adv.asp
BioMedcentral 